\title{
Rémy Leveau (Dir.), L’Algérie dans la guerre, Bruxelles, Ed. Complexe, 1995, 153 p
}

\section{Hamit Bozarslan}

\section{OpenEdition}

1 Journals

Édition électronique

URL : http://journals.openedition.org/conflits/503

DOI : $10.4000 /$ conflits.503

ISSN : $1777-5345$

Éditeur :

CCLS - Centre d'études sur les conflits lilberté et sécurité, L'Harmattan

Édition imprimée

Date de publication : 15 mai 1997

ISSN : 1157-996X

Référence électronique

Hamit Bozarslan, «Rémy Leveau (Dir.), L'Algérie dans la guerre, Bruxelles, Ed. Complexe, 1995, 153 p », Cultures \& Conflits [En ligne], 24-25 | hiver 1996- printemps 1997, mis en ligne le 27 mars 2007, consulté le 30 mars 2021. URL : http://journals.openedition.org/conflits/503 ; DOI : https://doi.org/ $10.4000 /$ conflits.503

Ce document a été généré automatiquement le 30 mars 2021.

Creative Commons License 


\title{
Rémy Leveau (Dir.), L’Algérie dans la guerre, Bruxelles, Ed. Complexe, 1995, $153 \mathrm{p}$
}

\author{
Hamit Bozarslan
}

1 Portant sur l'Algérie contemporaine cet ouvrage est dirigé par Rémy Leveau et regroupe six contributions : " Des crises à la guerre " (Rémy Leveau), " Une économie piégée entre trabendo et ajustement " (Omar Steele), " L'environnement de la violence : 'djihad' dans la banlieue d'Alger " (Luiz Martinez), " "Les héros n'ont pas de préoccupations de ce monde " " (Meriem Vergès), " Le FIS à l'épreuve de la lutte armée " (Séverine Labat) et finalement " Derrière la violence, la négociation " (Rémy Leveau).

2 L'Algérie dans la guerre respecte le cadre et la mission de la " Collection du CERI ": nombre limité de pages, manuel de base pour un grand public. II n'en est pas moins novateur en ce qu'il déplace l'analyse du discours idéologique (en l'occurrence islamisme) pour s'intéresser aux dynamiques politiques et sociales de l'Algérie en guerre et aux acteurs.

Dans sa première contribution, Rémy Leveau nous rappelle qu'au cours de la décennie 80 le régime algérien n'était pas plus menacé que d'autres régimes du monde musulman (Egypte, Tunisie notamment). II s'interroge alors sur les conditions économiques et sociales de cette irruption brusque de la guerre en Algérie. La situation économique s'aggrave à partir de 1986 (la chute du prix du pétrole réduit de moitié les ressources financières du pays) interdisant au régime la poursuite de la politique redistributive. Mais la crise financière n'explique pas tout. II convient également d'évoquer les manoeuvres malhabiles de la " classe dirigeante algérienne " qui crée " les conditions sociales, légales et politiques de sa mise à l'écart, elle la classe dirigeante refusera en fin de compte le changement, en ayant recours à l'armée, sans arriver pour autant à construire un schéma de sortie de crise ". Leveau analyse les émeutes urbaines de 1988. et souligne l'impact de la Guerre du Golfe, survenue à peine deux ans après, sur la vie politique algérienne. Cette guerre a eu deux conséquences contradictoires pour l'Algérie: elle a rappelé au gouvernement qu'il ne " disposait plus de contrepoids 
internationaux suffisants pour pouvoir prendre ses distances à l'égard l'Occident et faire preuve de solidarité avec l'Irak ", et elle a radicalisé la contestation. La Guerre du Golfe n'a pas seulement modifié le regard que l'opinion publique avait de l'Occident et de l'ancienne puissance colonisatrice, mais aussi la donne politique interne.

0. Steele nous livre une analyse de la situation économique. Son article rappelle naturellement les données économiques globales (un régime réticent aux réformes, mais qui perd dès les années 80 son monopole sur l'économie). Mais il est surtout novateur du fait qu'il analyse ce domaine ardu en termes d'acteurs. L'Algérie d'avant la guerre voit l'émergence de nouveaux types d'acteurs économiques, accomplissant cependant aussi un rôle social et politique important: les barons (descendants des grandes familles de commerçants et d'anciens officiers recyclés), les " managers " (jeunes cadres techniques et commerciaux), " les nouveaux venus " à la tête de petites entreprises et surtout : " les trebandistes ", ces jeunes qui parviennent à créer à la fois les moyens de survie et un espace de socialisation au sein de cette économie primitive. L'auteur conclut en soulignant les problèmes structurels de l'économie algérienne et la difficulté de mener des reformes dans une situation de guerre.

L'approche de L. Martinez est également très novatrice. Au-delà du mérite d'avoir mené des enquêtes dans une situation de violence, l'auteur montre que les dynamiques de la contestation armée ne s'expliquent que très marginalement par des arguments d'ordre théologique, voire idéologique. A la base, la contestation des banlieues et le rejet de la légitimité du pouvoir, rendent mentalement possible le passage à la violence : " les GIA et l'lAS représentent en 1994 des forces d'opposition légitimes pour une grande partie des jeunes des banlieues populaires ". En deuxième lieu, il insiste sur les pratiques de pouvoir de cette opposition, concrétisées selon un modèle fort ancien, kaïdale, mais avec des formes d'organisation et des modes de contrôle, de coercition et de cohésion tout à fait nouveaux. Ces pratiques ressemblent à celles que nous observons dans de nombreux cas de conflits : logique de racket (les " seigneurs " étant partis, privant ainsi les GIA de ressources importantes, ils sont obligés de " se rabattre sur les petits commerçants et entrepreneurs marginaux ") et de taxation contre protection. Des liens fort complexes s'établissent entre les délinquants et les émirs des GIA, et surtout entre ces derniers et les notables provinciaux. " Les notables, bien loin de dénoncer ou de combattre les maquisards, leur " achètent " la paix civile dans leur village ou leur bourg. Par exemple une partie des transports de marchandises qui viennent ravitailler les villages sont détournés par les maquisards qui prennent soin lorsqu'ils les arrêtent de ne pas détruire le véhicule ". Ce partage à l'amiable de ressources permet d'une part l'émergence d'une bourgeoisie dans les provinces algériennes, d'autre part assure aux GIA une persistance ancrée dans le local.

$6 \quad$ M. Verges part également d'études de terrain pour analyser l'aspect générationnel du conflit actuel en Algérie. La richesse de son étude réside dans sa capacité à rendre compte de la multiplicité des réponses à la crise ainsi que des rapports avec le système et la génération des parents. Si elle montre comment le FIS parvient, avec succès, à utiliser l'héritage de la lutte de libération nationale, elle n'en relève pas moins le scepticisme de nombreux jeunes à l'égard de Madani et de Belhadj. " L'absence d'une mémoire académique, regroupant et confrontant les mémoires des acteurs, écrit Verges, a amplifié la segmentation de l'espace des représentations et favorisé la cristallisation de mémoires plurielles ". La jeunesse pallie cette segmentation de la mémoire en inventant un âge d'or qu'elle n'a pas connu qui sert de justificatif à son 
action ] " L'impossibilité de " choisir " ses relations sociales, son activité professionnelle et son rang social remet à l'honneur un " avant " stable et rassurant, impliquant un coût très élevé ; " le changement " est dès lors perçu comme pénalisant " . Les jeunes en Algérie d'avant la guerre avaient l'impression de faire figure de " mauvaise herbe " . Cette auto-définition terrifiante explique largement l'opposition de la jeunesse au pouvoir, mais ajoutons-le- marque probablement aussi les limites de l'action du FIS et explique le succès actuel des GIA. La " mauvaise herbe " se replie sur son quartier, résistant par là même à toute logique centralisatrice d'un quelconque pouvoir et obéissant de nos jours localement à ses chefs de 20-25 ans.

7 S. Labat part de la chronologie de la guerre et remarque l'importance de l'attentat de l'aéroport d'Alger, (elle précise que de nombreux observateurs l'attribuent aux services de sécurité algériens) ainsi que du démantèlement des camps d'internement à partir de l'été 1993 dont les détenus reprennent en main les groupes armés isolés. Elle souligne la différence essentielle qui séparn le FIS des GIA : " Le FIS reste globalement décidé à articuler la violence qu'il administre à un projet politique concret et, fidèle aux attentes sociales de sa base, à continuer à incarner, malgré l'épreuve de la clandestinité l'alternative politique pour laquelle s'était prononcée une partie de la société algérienne au soir du 26 décembre 1991 ". Quant aux GIA, ils " rassemblent en effet une majorité d'éléments caractérisés par une commune explosion. Les GIA vont au demeurant permettre à des acteurs hétéronomes pour qui la violence va représenter la seule modalité d'intégration en ce qu'elle est créatrice de nouvelles identités et solidarités collectives, d'agréger ce que la diversité des motivations et des pratiques sociales, culturelles et politiques dissociait ". " Agés de moins de 21 ans, des mufti édictent les tables de la nouvelle loi, et autorisent des " émirs " du même âge à appliquer les " sentences " à l'encontre de ceux qui n'entendent pas se remettre à leur autorité nouvelle ". L'auteur insiste en fin sur la logique de guerre civile : la répression du pouvoir apporte une légitimation à la violence des groupes armés islamiques qui parviennent à nouer des relations de clientélisme à niveau local. Elle s'interroge sur l'avenir du FIS et sur les possibilités d'un dialogue avec le pouvoir. Toutefois, elle précise qu'une ré-institutionnalisation du FIS, même dans l'hypothèse de l'intégration d'une partie de la jeunesse révoltée ne suffirait pas pour en finir avec la violence, tant est profonde " la décomposition sociale et politique de la société algérienne ".

La dernière contribution, de Rémy Leveau, nous invite tout d'abord à dépasser le contexte actuel et à placer la guerre d'Algérie dans la durée, où l'islamisme ne pourrait être perçu comme une option religieuse contre le nationalisme : " on peut se demander si cette longue phase de violence n'apparaitra pas un jour, avec le recul, comme une étape de la lutte d'un nouveau courant nationaliste arabe, pour contrôler et moderniser la société arabe en écartant, au moins partiellement, la génération précédente après l'échec de son projet de construction nationale et d'affirmation face à l'occident ". Rémy Leveau insiste également sur l'hétérogénéité de la mouvance islamiste où deux logiques s'affrontent: " l'une pouvant s'intégrer dans une opération de reconstruction de l'Etat " l'autre " dérivant dans la société des milices " . Enfin, il s'interroge sur la possibilité d'une recomposition de l'espace politique algérien avec l'option d'intégration du FIS: " un pouvoir doté d'une légitimité nouvelle, incorporant une partie de l'opposition islamiste et ne refusant pas le choix des urnes pourra ramener la capacité de nuisance des GlA à un niveau minimal de type basque ou irlandais, sans commune mesure avec son intensité actuelle ". 
La conclusion signée par quatre des cinq collaborateurs de l'ouvrage ouvre des pistes de recherche extrêmement riches: les auteurs analysent les raisons sociologiques de la montée du FIS : " le principal succès de l'islamisme en Algérie tient à la jonction qu'il a réalisé dans sa phase légaliste, entre les élites et les classes populaires ". Cette capacité devient un atout majeur à l'heure de " l'illégitimité généralisée du pouvoir ", le FIS reste un courant nationaliste et peut se présenter comme une alternative pleinement algérienne. II hérite également de tout un vocabulaire de combat précédemment élaboré par le pouvoir : la fameuse hizb França qui dans les faits transpose la guerre contre l'ancien ennemi en un clivage intra-algérien (sans pour autant empêcher, précisons-le, le pouvoir d'utiliser ses relations privilégiées avec Paris comme une source de légitimité sur le plan intérieur), l'image des Harki etc..

Par ailleurs, les auteurs analysent l'engagement des jeunes sous un nouvel angle. L'émergence de l'individualisme, notamment chez eux, semble ainsi paradoxalement devenir un facteur déterminant de l'engagement politique, et au-delà, militaire : " on n'a pas voulu voir par exemple, qu'à travers l'islamisme, certains tentaient de briser le modèle patriarcal. Tout à coup, des jeunes garçons de 18 ans prenaient plus d'importance que leurs pères. Les jeunes gens sem !]laient se soumettre au modèle patriarcal pour mieux le subvertir, et les filles se soumettre au modèle islamique pour retrouver une liberté et échapper en partie au modèle autoritaire masculin " .

Plus généralement la conclusion présente une Algérie multiple et variée, dynamique malgré la guerre ] résistance des populations et lassitude de la violence y vont de pair. La société ne semble pas accepter les ordres venant des mufti et des émirs des GIA, mais sa lassitude est néanmoins évidente. Alors que le gouvernement compte sur ce sentiment, le FIS au contraire, tente de préserver ses relais et de garder un soutien dans la population.

Bien que la période qui nous séparent de la publication de cet ouvrage ait été riche en événements (rencontres de Saint Egidio, élections présidentielles, retour du FLN dans le giron du pouvoir sans pour autant rétablissement des anciens rapports de dépendance, condamnat on désormais explicite des GIA par le FIS et violence toujours aussi intense), ce petit ouvrage collectif reste d'une grande utilité. Les événements ont confirmé la plupart de ses analyses, rendant encore plus nécessaire la poursuite des démarches sociologiques qui en sont à la base.

\section{INDEX}

Mots-clés : guerre

Index géographique : Algérie, Maghreb 